\title{
How should we decide the optimum intraoperative fluid and colloid usage in pulmonary resection?
}

\author{
Yukio Sato \\ Department of General Thoracic Surgery, University of Tsukuba, Tsukuba, Japan \\ Correspondence to: Yukio Sato, MD, PhD. Department of General Thoracic Surgery, University of Tsukuba, 1-1-1 Tennodai, Tsukuba, Ibaraki 305- \\ 8575 Japan. Email: ysato@md.tsukuba.ac.jp. \\ Provenance: This is an invited article commissioned by the Academic Editor Dr. Shuangjiang Li (Department of Thoracic Surgery and West China \\ Medical Center, West China Hospital, Sichuan University, Chengdu, China). \\ Comment on: Wu Y, Yang R, Xu J, et al. Effects of Intraoperative Fluid Management on Postoperative Outcomes After Lobectomy. Ann Thorac Surg \\ 2019;107:1663-9.
}

Submitted Sep 30, 2019. Accepted for publication Oct 24, 2019.

doi: $10.21037 /$ jtd.2019.10.63

View this article a t: http://dx.doi.org/10.21037/jtd.2019.10.63

Lung cancer is the leading cause of cancer death throughout the world and pulmonary resection remains a major surgical procedure for the cure of localized non-small cell lung cancer. Respiratory complications are still the major cause of mortality and morbidity after lung resection. Pneumonia and acute respiratory distress syndrome (ARDS) are responsible for the great part of respiratory-related deaths after lung resection (1). Acute lung injury is the most frequent cause of mortality after lung resection (2), and a main purpose of the pulmonary anesthesiologist is to prevent this critical complication. Pulmonary edema following pneumonectomy was first reported in 1984 by Zeldin et al. (3) and they reported that excessive fluid administration causes pulmonary edema. Excessive fluid administration could also cause lung injury with lesser pulmonary resections (4). Currently, the incidence of lung injury following lung resection is approximately $3 \%$ with the mortality rate remains around $40 \%$ (5). Therefore, volume-restrictive fluid regime of $1-2 \mathrm{~mL} / \mathrm{kg} / \mathrm{h}$ has been proposed for intraoperative and postoperative periods. The purpose is to control the amount of fluid and reduce the hydrostatic pressure in the pulmonary microcirculation (6). However, such restrictive fluid management could cause impaired tissue perfusion due to dehydration, which leads to organ dysfunction represented by acute kidney injury (7). The aim of maintaining intraoperative euvolemia with a dry lung has been discussed repeatedly $(6,8)$, however a large randomised control study has not been conducted on the optimal fluid administration in pulmonary resection.

Therefore, identifying the causes leading to respiratory complications and the active prevention and reduction of their incidence has important clinical significance. In this study, Wu reported on the effects of intraoperative fluid management on postoperative outcomes after lobectomy, focusing on the total fluid infusion rate and colloid usage. They conducted a retrospective analysis of adult patients undergoing minimally invasive lobectomy. The primary exposure variables were intraoperative total fluid infusion rate and intraoperative colloid infusion rate. Compared with the moderate group $(9.5-11.8 \mathrm{~mL} / \mathrm{kg} / \mathrm{h})$ of intraoperative total fluid infusion rate, the risk for pulmonary complications was significantly increased at restrictive $(<9.5 \mathrm{~mL} / \mathrm{kg} / \mathrm{h})$, and liberal $(>11.8 \mathrm{~mL} / \mathrm{kg} / \mathrm{h})$ groups. Compared with the moderate group of intraoperative colloid infusion rate, the risk for pulmonary complications significantly increased at no colloid and restrictive groups. The current literature lacks investigations of the effect of intraoperative colloid on pulmonary complications. The colloid can keep the lung dry by increasing plasma colloid osmotic pressure and decreasing pulmonary edema, which may reduce pulmonary complications. Authors also revealed that intraoperative colloid infusion rate $>3.8 \mathrm{~mL} / \mathrm{kg} / \mathrm{h}$ was associated with a lower incidence of pulmonary complications. They concluded that in patients undergoing minimally invasive lobectomy the infusion rates of intraoperative total fluid and intraoperative colloid were all significantly associated 
with pulmonary complications. Both restrictive and liberal intraoperative fluid administration were related to adverse effects on postoperative outcomes.

It is quite reasonable to consider that there must be the most suitable usage of intraoperative fluid and colloid administration, and their results might propose the appropriate range of fluid and colloid usage. However, the infusion rate that showed the less pulmonary complications is different from previous reports which recommended much smaller amount of fluid administration $(2,6)$. Although the differences in surgical procedures and anesthetic method might have affected, the difference in recommended dose of intraoperative fluid is quite significant. Authors speculated that the existence of the differences in intraoperative fluid volume and colloid volume are largely due to the different habits of the different anesthesiologists. However, it is possible that anesthesiologists adjusted the administrations of fluid and colloid depends on the conditions of the patients, such as cardiac function, pulmonary function and renal function. Actually, the amount of intraoperative bleeding, which influences the amount of intraoperative fluid and postoperative course, was different by the study groups showed with supplemental data. In other words, the differences in fluid and colloid could be caused by the background conditions and intraoperative course of the patients, and obtained results might be biased. Therefore, a prospective randomized trial with several different volumes of fluid and colloid, including the recommended volumes by previous studies, expected to be performed to eliminate the bias such as pre-existing comorbidities of the patients and the intraoperative incidences. It is highly expected that the study will elucidate the optimum usage of intraoperative fluid and colloid.

\section{Acknowledgments}

None.

Cite this article as: Sato Y. How should we decide the optimum intraoperative fluid and colloid usage in pulmonary resection? J Thorac Dis 2019;11(11):E204-E205. doi:10.21037/ jtd.2019.10.63

\section{Footnote}

Conflicts of Interest: The author has no conflicts of interest to declare.

Ethical Statement: The author is accountable for all aspects of the work in ensuring that questions related to the accuracy or integrity of any part of the work are appropriately investigated and resolved.

\section{References}

1. Dulu A, Pastores SM, Park B, et al. Prevalence and mortality of acute lung injury and ARDS after lung resection. Chest 2006;130:73-8.

2. Licker $M$, Fauconnet $P$, Villiger $Y$, et al. Acute lung injury and outcomes after thoracic surgery. Curr Opin Anaesthesiol 2009;22:61-7.

3. Zeldin RA, Normandin D, Landtwing D, et al. Postpneumonectomy pulmonary edema. J Thorac Cardiovasc Surg 1984;87:359-65.

4. Chau EH, Slinger P. Perioperative fluid management for pulmonary resection surgery and esophagectomy. Semin Cardiothorac Vasc Anesth 2014;18:36-44.

5. Licker M, de Perrot M, Spiliopoulos A, et al. Risk factors for acute lung injury after thoracic surgery for lung cancer. Anesth Analg 2003;97:1558-65.

6. Evans RG, Naidu B. Does a conservative fluid management strategy in the perioperative management of lung resection patients reduce the risk of acute lung injury? Interact Cardiovasc Thorac Surg 2012;15:498-504.

7. Ahn HJ, Kim JA, Lee AR, et al. The Risk of Acute Kidney Injury from Fluid Restriction and Hydroxyethyl Starch in Thoracic Surgery. Anesth Analg 2016;122:186-93.

8. Assaad S, Popescu W, Perrino A. Fluid management in thoracic surgery. Curr Opin Anaesthesiol 2013;26:31-9. 